\title{
Determinantes clínicos en la memoria autobiográfica de pacientes con epilepsia del lóbulo temporal unilateral ${ }^{*}$
}

Clinical determinants of autobiographical memory in patients with unilateral temporal lobe epilepsy

\author{
DOI: $10.11144 / J a v e r i a n a . u p s y 15-5 . d c m a$ \\ Recepción: 17 Noviembre 2015 | Aprobación: 16 Noviembre 2016
}

\author{
Julián Carvajal-Castrillón \\ Instituto Neurológico de Colombia, Colombia \\ Daniel Camilo Aguirre-Acevedo \\ Universidad de Antioquia, Colombia \\ David Andrés Montoya Arenas \\ Universidad de San Buenaventura, Colombia
}

Para citar este artículo: Carvajal-Castrillón, J., AguirreAcevedo, D. C., \& Montoya Arenas, D. A. (2016). Determinantes clínicos en la memoria autobiográfica de pacientes con epilepsia del lóbulo temporal unilateral Universitas Psychologica, 15(5). http:// dx.doi.org/10.11144/Javeriana.upsy15-5.dcma

\section{RESUMEN}

El paciente con epilepsia del lóbulo temporal presenta generalmente una enfermedad de difícil manejo, la cual cursa con alteraciones en memoria autobiográfica. Sin embargo, la epilepsia es una enfermedad en la que actúan diversos factores clínicos que contribuyen a determinar la severidad y el pronóstico. El objetivo del artículo fue explorar la posible relación entre el rendimiento de los pacientes con epilepsia del lóbulo temporal con esclerosis hipocampal en memoria autobiográfica y las variables clínicas asociadas. La muestra estuvo conformada por 25 pacientes con diagnóstico de epilepsia del lóbulo temporal. Se les suministró la Entrevista de Memoria Autobiográfica para valorar el desempeño en memoria personal semántica e incidentes autobiográficos. Se determinó la relación entre el rendimiento en memoria autobiográfica y variables clínicas asociadas como edad de inicio, número de años con epilepsia, lateralización de la zona de inicio ictal, tipo de terapia farmacológica y número de medicamentos. Para determinar las correlaciones se calculó el coeficiente de correlación de Spearman, mientras que para la comparación entre grupos se utilizó la prueba U de Mann-Whitney. Variables clínicas como el tiempo de la evolución de la enfermedad, empleo de politerapia y número de medicamentos, afectan el desempeño en memoria autobiográfica en pacientes con epilepsia del lóbulo temporal. Si bien, la epilepsia del lóbulo temporal genera alteraciones en la memoria autobiográfica, diversas variables clínicas asociadas a la enfermedad, afectan aún más la capacidad mnésica de los pacientes.

Palabras clave

Epilepsia del lóbulo temporal; Memoria autobiográfica; Neuropsicología.

\begin{abstract}
The patient with temporal lobe epilepsy generally has a difficult to control disease, which develops with alterations in autobiographical memory. However, epilepsy is a disease with various clinical factors acting, which help determine the severity and prognosis. The aim of this study was to explore the possible relationship between the performance
\end{abstract}


of patients with temporal lobe epilepsy and hippocampal sclerosis in autobiographical memory and associated clinical variables. The sample was composed by 25 patients diagnosed with temporal lobe epilepsy. They were supplied with the Autobiographical Memory Interview to assess both the performance in personal semantic memory and autobiographical incidents. The relationship between autobiographical memory performance and associated clinical variables as age of onset, number of years with epilepsy, lateralization of ictal onset zone, drug resistance, type of drug therapy and number of antiepileptic drugs was determined. A Spearman Rho Correlation Coefficient was calculated to measure the correlations between $A$ and $B$, while Mann-Whitney $U$ test was used to establish between-group differences. Clinical variables such as time of evolution of the disease, polytherapy use and number of antiepileptic drugs, affect autobiographical memory performance in patients with temporal lobe epilepsy. While the temporal lobe epilepsy generates changes in autobiographical memory, various clinical variables associated with the disease, affects even more the patients' memory.

Keywords

Autobiographical Memory; Temporal lobe epilepsy;

Neuropsychology.

\section{Introducción}

El paciente con epilepsia del lóbulo temporal (ELT) presenta generalmente una enfermedad de difícil manejo (Cendes, 2004; Mohanraj \& Brodie, 2006; Wiebe, 2000), y cursa con alteraciones en el funcionamiento cognitivo, específicamente trastornos de la memoria (Keary, Frazier, Busch, Kubu, \& Iampietro, 2007; Sheth, 2002). La memoria episódica se relaciona con el recuerdo de las situaciones en general, tanto en las que la persona ha tenido un papel únicamente expectante, como aquellos sucesos en los que participa de manera activa y se involucra afectivamente (Carrillo-Mora, 2010). Este último tipo de proceso se denomina memoria autobiográfica (MA), y está compuesta por todos los eventos que una persona experimenta y, por ende, le permite crear un sentimiento de identidad (Marín \& Ruiz, 2011). En estudios anteriores, se ha encontrado que los pacientes con ELT presentan alteraciones en la MA, principalmente en el recuerdo de incidentes autobiográficos y en especial, en los casos de zona de inicio ictal (ZII) mesial (Carvajal-Castrillón,
Andrade, Aguirre-Acevedo \& Montoya, 2016; Múnera et al, 2014).

Sin embargo, la epilepsia es una enfermedad en la que actúan diversos factores clínicos que contribuyen a determinar el cuadro clínico del paciente, influyendo en la severidad y el pronóstico de los trastornos mnésicos que el paciente puede presentar, lo que provoca un mayor nivel de discapacidad en sus actividades cotidianas (Gregory, Nenert, Allendorfer, Martin, Kana, \& Szaflarski, 2015). Variables como la edad de inicio de las crisis, tiempo de evolución de la enfermedad, la lateralización de la ZII y el tratamiento farmacológico implementado, se han relacionado en algunos estudios con diferencias en las alteraciones cognitivas, incluyendo un déficit en MA (Helmstaedter, Elger, \& Witt, 2016). En la actualidad, aún no hay resultados claros y homogéneos acerca de la influencia de estos determinantes clínicos en el desempeño en MA en los pacientes con ELT (Carvajal-Castrillón \& Montoya, 2015). Es por esto, que el objetivo del estudio fue explorar la correlación de las variables clínicas con la MA, en pacientes con ELT.

\section{Método y procedimiento}

\section{Pacientes}

Muestreo no probabilístico, considerando a los pacientes con epilepsia que consultaron entre 2013-2015 al Grupo de Epilepsia de Difícil Control del Instituto Neurológico de Colombia (INDEC). La muestra estuvo conformada por 25 participantes con diagnóstico electroclínico de ELT según los criterios de la ILAE (Beghi et al, 2005) y presencia de esclerosis hipocampal (EH) según estudio de RMN con resonador de 1.5 tesla (Araújo et al, 2006; Beghi et al, 2005; Nunes et al 2011; Uscátegui \& Izquierdo, 2003).

\section{Procedimiento}

Se revisó la historia clínica electrónica de pacientes que habían sido diagnosticados por 
el Grupo de Epilepsia del INDEC con ELT unilateral del INDEC y que cumplían con los criterios de inclusión. Luego, se concertó con el paciente una sesión de noventa minutos en la mañana, para realizar la evaluación de la MA, la cual la realizó un psicólogo con entrenamiento previo en el test en el INDEC. Al final, se organizó una base de datos con las variables demográficas y de análisis. De todos los participantes se obtuvo un consentimiento informado, aprobado por los Comités de Bioética de Universidad de San Buenaventura e INDEC. Se realizó un estudio correlacional con diseño transversal.

\section{Criterios de inclusión}

Pacientes entre los 25 y 50 años con diagnóstico de ELT unilateral. Ninguno había sido sometido a cirugía de epilepsia. Se excluyeron sujetos con coeficiente intelectual inferior a 80, según la Escala de Inteligencia de Wechsler para adultos WAIS III (Wechsler, 1997). De igual forma, fueron excluidos pacientes con otras enfermedades neurológicas o trastornos psiquiátricos no tratados al momento de la evaluación, empleando la Mini Entrevista Neuropsiquiátrica Internacional (MINI) (Lecrubier, 1998). Tampoco se consideraron pacientes que habían recibido topiramato o tenían antecedentes tóxicos.

\section{Variables de análisis}

Se consideró la edad, sexo y escolaridad (número de años académicos aprobados) como variables sociodemográficas. Las variables de análisis fueron el desempeño en memoria personal semántica e incidentes autobiográficas en la Entrevista de Memoria Autobiográfica (EMA), así como su correlación con la edad de inicio de las crisis, tiempo de evolución de la enfermedad, lateralización de la ZII, tipo de terapia farmacológica y número de medicamentos.

\section{Conformación de los grupos}

Se conformaron grupos para su comparación, según la lateralidad de la ZII y el tipo de tratamiento. Según estudio de videomonitoreo electroencefalográfico, fueron conformados dos grupos: 13 pacientes con ZII derecha y 12 pacientes con ZII izquierda. Según el tipo de tratamiento farmacológico recibido, se establecieron dos grupos: monoterapia $(n=5)$ o politerapia $(n=20)$.

\section{Evaluación de la memoria autobiográfica}

La Entrevista de Memoria Autobiográfica EMA, es un cuestionario semi-estructurado, que se divide en dos partes: información personal semántica e incidentes autobiográficos. Las preguntas se distribuyen en tres diferentes periodos de la vida del evaluado: infancia (de los 0 a los 18 años), adultez temprana (de los 19 a los 30 años) y vida reciente (últimos 5 años). La memoria semántica personal está compuesta por datos e información como fechas y nombres de personas y de lugares relevantes en la vida del paciente (Carrillo-Mora, 2010). Para evaluar este tipo de memoria, se utilizan preguntas como "diga el nombre de tres profesores y amigos de la escuela". Mientras que los incidentes autobiográficos son todos sucesos en los que el sujeto participa de manera activa y se involucra afectivamente (Marín \& Ruiz, 2011); para evaluarlos se emplean preguntas como "mencione algún incidente que involucre a un pariente o visitante en el último año". En cuanto a la calificación, la puntuación de cada ítem o pregunta varía entre $0-3$, y al final de la prueba se califica cada periodo de la vida por separado. La puntuación para la información personal semántica en cada uno de los tres periodos varía entre 0-21, para una puntuación total máxima de 63. Los incidentes autobiográficos para cada uno de los tres periodos de la vida, varía entre $0-9$, para una puntuación total máxima de 21. Para este estudio se empleó la versión original de la EMA validada en idioma 
español (García, Contador, Fernández-Calvo \& Ramos, 1989).

\section{Análisis de los datos}

Se describieron las características demográficas, clínicas y el rendimiento en las subpruebas de MA entre los pacientes con ELT por EH según lateralización de la zona de inicio ictal y tipo de terapia farmacológica. Para las comparaciones intragrupo en memoria personal semántica y recuerdo de incidentes autobiográficos, se utilizó la prueba U de Mann Whitney y se calculó el tamaño del efecto no paramétrico (TE) (Fritz, Morris \& Richler, 2012) para el cual se asumió una diferencia importante entre los grupos si TE $>0.5$. Este TE se calculó mediante la fórmula: $\mathrm{TE}=\mathrm{Z} / \sqrt{ } \mathrm{N}$ ); donde $\mathrm{Z}=$ es el estadístico $z$ de la aproximación asintótica de la prueba $U$ de Mann-Whitney; $\mathrm{N}$ es el tamaño de muestra total (Larner, 2014). Se exploró la correlación entre el rendimiento de las dimensiones de la MA con las variables de análisis clínico como la edad de inicio de las crisis, tiempo de evolución de la epilepsia y número de medicamentos, calculando el coeficiente de correlación de Spearman. Para los análisis estadísticos se utilizó el software IBM SPSS statistics versión 22. Se empleó como nivel de significación estadístico para el control del error tipo I \#=0.05.

\section{Resultados}

\section{Características demográficas y clínicas}

La mayoría de participantes eran de sexo femenino, la mediana de la edad fue de 44 años y de escolaridad de 11 años. El coeficiente intelectual se ubicó en rango normal-bajo. El $80 \%$ de los sujetos de la muestra habían sido tratados con politerapia. En cuanto al número de medicamentos, el $48 \%$ de los participantes recibían dos medicamentos. La mediana de edad de inicio de las crisis fue a los 12 años, y del número de años con epilepsia fue equivalente a 28. En el $52 \%$ de los participantes se identificó una $\mathrm{EH}$ derecha mediante estudio de RMN, con zona de inicio ictal ipsilateral (Tabla 1)

\section{TABLA 1}

\begin{tabular}{|l|l|}
\hline & $\begin{array}{l}\text { ELT por EH } \\
n=25\end{array}$ \\
\hline Sexo. Frecuencia (\%) & \\
\hline Femenino & $19,0(76,0)$ \\
\hline Masculino & $6,0(24,0)$ \\
\hline Edad en anios. Mediana (RI) & $44,0(10,5)$ \\
\hline Escolaridad en años. Mediana (RI) & $11,0(5,0)$ \\
\hline Coeficiente intelectual. Mediana (RI) & $84,0(7,0)$ \\
\hline Edad de inicio de las crisis. Mediana (RI) & $12,0(12,5)$ \\
\hline Número de años con epilepsia. Mediana (RI) & $28,0(19,0)$ \\
\hline Esclerosis hipocampal (\%) & \\
\hline Derecha & $13(52)$ \\
\hline Izquierda & $12(48)$ \\
\hline Zona de inicio ictal (\%) & \\
\hline Derecha & $13(52)$ \\
\hline Izquierda & $12(48)$ \\
\hline Refractariedad al tratamiento (\%) & \\
\hline Refractario & $22(88)$ \\
\hline No refractario & $3(12)$ \\
\hline Tratamiento farmacologico (\%) & \\
\hline Monoterapia & $5(20)$ \\
\hline Politerapia & $20(80)$ \\
\hline Número de medicamentos (\%) & $5(20)$ \\
\hline Un medicamento & $12(48)$ \\
\hline Dos medicamentos & $8(32)$ \\
\hline Tres medicamentos & \\
\hline
\end{tabular}

RI: Rango intercuartil (Percentil 75-Percentil25) Fuente: elaboración propia

Edad de inicio de las crisis y memoria autobiográfica

Para la memoria semántica personal, no se encontró correlación. Tampoco se observó un mayor déficit en el recuerdo de incidentes autobiográficos en los pacientes con edad de inicio más temprana

Tiempo de evolución de la epilepsia y memoria autobiográfica

Se encontró correlación entre el número de años con epilepsia y la memoria semántica de los periodos previo a la escuela $(-0.421)$ y de la infancia (-0.429). En cuanto al recuerdo de incidentes autobiográficos, no se encontró que los pacientes con mayor número de años 
de evolución de la enfermedad, tuvieran más alteraciones en este tipo de memoria

\section{Lateralización de la zona de inicio ictal y memoria autobiográfica}

Teniendo en cuenta el tamaño del efecto $(>0.5)$ $\mathrm{y}$ el valor $\mathrm{p}(<0.05)$, no se encontraron diferencias estadísticamente significativas entre los grupos en memoria semántica personal ni en incidentes autobiográficos (ver Tabla 2).

\section{TABLA 2}

\begin{tabular}{|c|c|c|c|c|c|}
\hline & $\begin{array}{l}\text { Pacientes con ZII } \\
\text { derecha } \\
n=13\end{array}$ & $\begin{array}{l}\text { Pacientes con ZII } \\
\text { izquierda } \\
\mathrm{n}=12\end{array}$ & TE & $\mathrm{z}$ & Valor $\mathrm{p}$ \\
\hline Semántica antes de la escuela & $3,0(2,8)$ & $4,8(1,5)$ & 0,3 & $-1,4$ & 0,154 \\
\hline Semántica escuela & $6,5(2,0)$ & $7,0(1,4)$ & 0,3 & $-1,4$ & 0,157 \\
\hline Semántica bachillerato & $7,5(1,3)$ & $7,0(1,3)$ & 0,2 & $-0,9$ & 0,349 \\
\hline Semántica infancia & $17,0(3,3)$ & $18,3(2,4)$ & 0,3 & $-1,4$ & 0,148 \\
\hline Semántica carrera & $7,5(1,5)$ & $7,0(1,3)$ & 0,1 & $-0,3$ & 0,780 \\
\hline Semántica boda & $8,0(2,0)$ & $8,0(1,0)$ & 0,1 & $-0,7$ & 0,474 \\
\hline Semántica hijos & $4,0(0,0)$ & $3,5(0,5)$ & 0,4 & $-2,0$ & $0,041^{*}$ \\
\hline Semántica adultez & $19,0(2,3)$ & $18,5(0,5)$ & 0,0 & $-0,1$ & 0,910 \\
\hline Semántica hospital actual & $6,0(1,3)$ & $6,0(1,3)$ & 0,0 & $-0,2$ & 0,845 \\
\hline Semántica hospital previo & $7,0(1,0)$ & $6,8(1,6)$ & 0,2 & $-1,2$ & 0,224 \\
\hline Semántica navidad & $2,0(0,0)$ & $2,0(0,0)$ & 0,2 & $-1,0$ & 0,298 \\
\hline Semántica vacaciones & $3,0(0,5)$ & $3,0(0,0)$ & 0,3 & $-1,6$ & 0,109 \\
\hline Semántica vida reciente & $18,0(1,5)$ & $17,5(2,0)$ & 0,1 & $-0,3$ & 0,783 \\
\hline SEMANTICA TOTAL & $53,0(6,3)$ & $53,8(4,9)$ & 0,2 & $-1,1$ & 0,276 \\
\hline $\begin{array}{l}\text { Autobiográfica antes de la } \\
\text { escuela }\end{array}$ & $1,0(1,5)$ & $1,5(1,0)$ & 0,2 & $-0,8$ & 0,408 \\
\hline Autobiográfica escuela & $1,0(1,0)$ & $1,0(1,0)$ & 0,1 & $-0,4$ & 0,708 \\
\hline Autobiografica bachillerato & $1,0(1,0)$ & $1,0(0,8)$ & 0,0 & $-0,2$ & 0,862 \\
\hline Autobiográfica infancia & $3,0(2,0)$ & $4,0(1,8)$ & 0,1 & $-0,7$ & 0,486 \\
\hline Autobiográfica carrera & $1,0(1,0)$ & $1,0(0,8)$ & 0,2 & $-0,8$ & 0,423 \\
\hline Autobiografica boda & $1,0(1,0)$ & $1,0(1,0)$ & 0,0 & $-0,2$ & 0,825 \\
\hline Autobiográfica hijos & $2,0(1,0)$ & $1,0(0,8)$ & 0,3 & $-1,3$ & 0,198 \\
\hline Autobiográfica adultez & $4,0(1,0)$ & $4,0(0,0)$ & 0,4 & $-2,2$ & $0,030^{*}$ \\
\hline Autobiografica hospital actual & $2,0(1,5)$ & $2,0(0,8)$ & 0,2 & $-0,9$ & 0,379 \\
\hline Autobiográfica hospital previo & $2,0(0,5)$ & $2,0(0,8)$ & 0,1 & $-0,4$ & 0,677 \\
\hline Autobiográfica vacaciones & $2,0(0,0)$ & $2,0(0,0)$ & 0,0 & $-0,0$ & 0,971 \\
\hline Autobiográfica vida reciente & $6,0(1,5)$ & $6,0(1,8)$ & 0,2 & $-0,8$ & 0,438 \\
\hline AUTOBIOGRAFICA TOTAL & $14,0(2,0)$ & $13,5(2,5)$ & 0,2 & $-1,1$ & 0,277 \\
\hline
\end{tabular}

ZII: zona de inicio ictal. M: Mediana. Ri: Rango intercuartil (Percentil 75 -Percentil25. TE:

Tamaño del efecto; $z$ : Estadístico z aproximación asintónica de la prueba U de Mann Whitney.

* Valor estadísticamente significativo Fuente: elaboración propia

Tipo de tratamiento farmacológico y memoria autobiográfica

En memoria semántica personal, los pacientes intervenidos con politerapia, tuvieron un desempeño significativamente inferior en el recuerdo de información semántica de la escuela. En incidentes autobiográficos, no se encontraron diferencias estadísticamente significativas entre los grupos (Tabla 3).

\section{TABLA 3}

\begin{tabular}{|c|c|c|c|c|c|}
\hline & $\begin{array}{l}\text { Pacientes con } \\
\text { monoterapia } \\
n=5\end{array}$ & $\begin{array}{l}\text { Pacientes con } \\
\text { politerapia } \\
n=20\end{array}$ & $\mathrm{TE}$ & $z$ & Valor $\mathrm{p}$ \\
\hline $\begin{array}{l}\text { Semántica antes de la } \\
\text { escuela }\end{array}$ & $3,5(2,3)$ & $3,8(2,5)$ & 0,0 & -0.2 & 0,834 \\
\hline Semántica escuela & $8,0(0,3)$ & $6,8(1,0)$ & $0,6^{*}$ & $-2,8$ & $0,005^{*}$ \\
\hline Semántica bachillerato & $8,0(1,3)$ & $7,0(1,4)$ & 0,2 & $-1,0$ & 0,339 \\
\hline Semántica infancia & $19,5(2,3)$ & $17,5(2,8)$ & 0,4 & $-2,0$ & $0,048^{*}$ \\
\hline Semántica carrera & $7,5(1,8)$ & $7,0(1,4)$ & 0.1 & $-0,5$ & 0,601 \\
\hline Semántica boda & $9,0(1,5)$ & $8,0(2,0)$ & 0,2 & $-1,0$ & 0,334 \\
\hline Semántica hijos & $4,0(0,5)$ & $4,0(0,5)$ & 0,0 & $-0,0$ & 0,968 \\
\hline Semántica adultez & $18,5(2,3)$ & $18,8(0,5)$ & 0,1 & $-0,4$ & 0,697 \\
\hline Semántica hospital actual & $6,5(1,8)$ & $6,0(0,9)$ & 0,4 & $-2,2$ & $0,025 *$ \\
\hline Semántica hospital previo & $7,0(0.0)$ & $6,8(1,8)$ & 0,3 & $-1,3$ & 0,206 \\
\hline Semántica navidad & $2,0(0,0)$ & $2,0(0,0)$ & 0,1 & $-0,5$ & 0,617 \\
\hline Semántica vacaciones & $3,0(0.3)$ & $3,0(0,4)$ & 0,1 & $-0,4$ & 0,716 \\
\hline Semántica vida reciente & $18,5(2,0)$ & $17,5(1,8)$ & 0,5 & $-2,3$ & $0,021^{*}$ \\
\hline SEMÁNTICA TOTAL & $58,0(5,3)$ & $53,0(4,1)$ & 0,5 & $-2,5$ & $0,014^{*}$ \\
\hline $\begin{array}{l}\text { Autobiográfica antes de la } \\
\text { escuela }\end{array}$ & $1,0(1,5)$ & $1,0(1,0)$ & 0,1 & $-0,3$ & 0,796 \\
\hline Autobiográfica escuela & $2,0(1,0)$ & $1,0(1,0)$ & 0,2 & $-1,1$ & 0,293 \\
\hline Autobiográfica bachillerato & $1,0(1,0)$ & $1,0(0,8)$ & 0,1 & $-0,6$ & 0,573 \\
\hline Autobiográfica infancia & $5,0(2,0)$ & $4,0(2,0)$ & 0,1 & $-0,7$ & 0,514 \\
\hline Autobiográfica carrera & $1,0(1,0)$ & $1,0(1,0)$ & 0,1 & $-0,3$ & 0,739 \\
\hline Autobiográfica boda & $2,0(0,5)$ & $1,0(1,0)$ & 0,4 & $-1,8$ & 0,076 \\
\hline Autobiográfica hijos & $1,0(1,0)$ & $1,0(1,0)$ & 0,1 & $-0,7$ & 0,505 \\
\hline Autobiográfica adultez & $4,0(1,5)$ & $4,0(0,8)$ & 0,2 & $-1,0$ & 0,340 \\
\hline $\begin{array}{l}\text { Autobiográfica hospital } \\
\text { actual }\end{array}$ & $2,0(1,0)$ & $2,0(1,0)$ & 0,3 & $-1,6$ & 0,103 \\
\hline $\begin{array}{l}\text { Autobiográfica hospital } \\
\text { previo }\end{array}$ & $2,0(0,0)$ & $2,0(1,0)$ & 0,2 & $-1,1$ & 0,278 \\
\hline Autobiográfica vacaciones & $2,0(0,0)$ & $2,0(0,0)$ & 0,1 & $-0,5$ & 0,649 \\
\hline $\begin{array}{l}\text { Autobiográfica vida } \\
\text { reciente }\end{array}$ & $6,0(1,0)$ & $6,0(1,0)$ & 0,4 & $-1,3$ & $0,062^{*}$ \\
\hline AUTOBIOGRÁFICA TOTAL & $15,0(2,5)$ & $13,5(1,8)$ & 0,4 & $-2,1$ & $0,034^{*}$ \\
\hline
\end{tabular}

ZII: zona de inicio ictal. M: Mediana. RI: Rango intercuartil (Percentil75-Percentil25). TE:Tamaño del efecto; z: Estadístico z aproximación asintótica de la prueba U de Mann Whitney.

*Valor estadísticamente significativo Fuente: elaboración propia

Número de medicamentos y memoria autobiográfica

En memoria semántica personal, se encontró correlación entre el número de medicamentos y el recuerdo de información de los periodos de la escuela $(-0.400)$, la boda $(-0.536)$ y la vida reciente $(-0.431)$, así como en la puntuación de la memoria semántica personal total $(-0.532)$. De igual manera, se encontró correlación entre esta variable y el recuerdo de incidentes autobiográficos de los periodos hospital previo $(-0.417)$, hospital actual $(-0.411)$ y vida reciente 
(-0.596), así como con la puntuación total de incidentes autobiográficos $(-0.594)$.

\section{Discusión}

El objetivo del estudio fue explorar la correlación de la MA con variables clínicas asociadas, en pacientes con ELT. No se encontró correlación entre el tiempo de evolución de la enfermedad y el recuerdo de incidentes autobiográficos, pero sí con la memoria personal semántica para los periodos previos a la escuela e infancia. El periodo de la infancia alude a los recuerdos del sujeto desde el inicio de su vida hasta los 18 años de edad. Este resultado sugiere que a mayor tiempo de evolución de la enfermedad, peor es el rendimiento de los pacientes con ELT por EH en la memoria personal semántica de esta época de la vida.

Estudios previos en pacientes con ELT han demostrado la correlación entre el tiempo de evolución de la epilepsia y una alteración difusa en las habilidades cognitivas del paciente (Cormack et, 2007). Sin embargo, la correlación entre esta variable clínica y el bajo rendimiento en pruebas de MA no ha sido aún demostrada (Carvajal-Castrillón \& Montoya, 2015). A pesar de que en las investigaciones sobre la MA en la ELT no se ha reportado dicha correlación, es algo que se puede esperar, teniendo en cuenta que la ELT es una enfermedad de difícil manejo (Mohanraj \& Brodie, 2006) y su curso crónico genera un deterioro cognitivo progresivo (Feria-Romero et al, 2013), razón por la cual, la epilepsia puede ser considerada como una pseudodemencia en la que predominan alteraciones mnésicas (Kambaja \& Dupont, 2016), estableciéndose posibles asociaciones entre el déficit cognitivo y la frecuencia ictal (Voltzenlogel, Vignal, Hirsch \& Manning, 2014).

Respecto a la relación entre el tratamiento farmacológico y la MA en pacientes con ELT, se correlacionó el desempeño en memoria personal semántica y el recuerdo de incidentes autobiográficos, con el tipo de tratamiento farmacológico. Para el recuerdo de incidentes autobiográficos no se encontraron diferencias entre los pacientes con monoterapia comparados con pacientes que recibieron politerapia. Sin embargo, se reportaron diferencia entre ambos grupos en la memoria personal semántica de la escuela, siendo peor el desempeño en los pacientes con politerapia.

En la literatura se han reportado los efectos cognitivos de los medicamentos antiepilépticos en procesos mentales superiores como atención, memoria, funcionamiento ejecutivo y lenguaje (Mula \& Trimble, 2006). Particularmente, en los estudios de ELT y MA, también se ha encontrado diferencias en el desempeño al comparar pacientes con monoterapia y politerapia. Lah, Lee, Garyson y Miller (2006), encontraron en su estudio, como los pacientes con politerapia tenían un peor rendimiento en el recuerdo de incidentes autobiográficos. Helmstaedter, Elger, y Witt (2016), reportaron también problemas en la memoria episódica según las dosis de medicamentos empleados en epilepsia farmacorresistente.

En nuestro estudio también se analizó la correlación entre el número de medicamentos y la MA, ya que en anteriores estudios sobre la neuropsicología de la epilepsia, se ha reportado una relación entre el número de medicamentos y la gravedad del deterioro cognitivo (Mula \& Trimble, 2009). En memoria semántica personal, se encontró correlación entre el número de medicamentos y el recuerdo de información de los periodos de la escuela, la boda y la vida reciente, así como en la puntuación de la memoria semántica personal total. Además, se encontró correlación entre esta variable y el recuerdo de incidentes autobiográficos de los periodos hospital previo y hospital actual y vida reciente, así como con la puntuación total de incidentes autobiográficos. Similares resultados fueron reportados en un estudio de Lah, Lee, Garyson y Miller, 2006, en el cual los pacientes que recibían más de dos medicamentos, tenían un peor rendimiento en la evocación de recuerdos autobiográficos. La investigación de Helmstaedter, Elger, y Witt (2016), confirmó este hallazgo.

Como siguiente punto de discusión, se analizó la relación entre la lateralización de la ZII 
con el desempeño de los pacientes en MA. No se encontraron diferencias en ninguna de las variables de la MA ambos grupos de pacientes, puesto que, tanto los pacientes con ZII izquierda como derecha, presentaron una marcada alteración en el recuerdo de incidentes autobiográficos.

Respecto a la influencia de la lateralización de la ZII y la MA, los resultados en la literatura son discordantes. Carvajal-Castrillón, Andrade, Aguirre-Acevedo y Montoya, 2016, reportaron alteraciones en la MA con igual severidad tanto en pacientes con ZII izquierda como derecha. Por su parte, Voltzenlogel, Després y Vignal (2006), encontraron un rendimiento significativamente inferior en MA en los pacientes con ZII izquierda. De igual forma, para Lah, Lee, Garyson y Miller, 2006, los pacientes con ZII izquierda presentan un peor desempeño en todas las pruebas de memoria remota; además, se observó en su estudio como los pacientes con ZII derecha presentan un déficit específico para el recuerdo de incidentes autobiográficos, pero no para la memoria personal semántica, tal como se evidencia en el estudio de Múnera et al (2014). En el estudio de Herfur, Kasper, Schwarz, Stefan y Pauli (2010), se concluyó que los pacientes con ELT mesial presentaron un pobre rendimiento en recuerdo de incidentes autobiográficos, independiente de la lateralización de la ZII; sin embargo se observó un peor desempeño en memoria personal semántica en los pacientes con ZII izquierda. Estudios como el de St-Lauren, Moscovitch, Levine y McAndrews, 2009, apoyan la afirmación de que el hipocampo izquierdo es más relevante en el recuerdo de información autobiográfica (Butler, Kapur, Zeman, Weller \& Connelly, 2012).

En cuanto a la relación entre lateralización ZII y memoria personal semántica, es posible afirmar que en pacientes con ELT por EH no hay ninguna relación entre ambas variables, ya que la memoria personal semántica depende de áreas temporales laterales (Antérion, Mazzola \& Laurent, 2008) $\mathrm{y}$ puede funcionar adecuadamente aun cuando hay un daño hipocampal (Carvajal-Castrillón \&
Montoya, 2015; Nadel, Samsonovich, Ryan \& Moscovitch, 2000).

Respecto a la relación entre el recuerdo de incidentes autobiográficos y la lateralización de la ZII, en este estudio no fue demostrada, ya que se observó un marcado déficit en este tipo de memoria, tanto en pacientes con ZII izquierda como derecha. Este hallazgo se explicaría por el patrón de diseminación de la actividad ictal, según el cual, la actividad eléctrica iniciada en un hipocampo tiende a diseminarse a las regiones contralaterales, generando así una disfunción tanto en el hipocampo lesionado como en el hipocampo contralateral (Pfeuty, Thivard, Dupont, Adam, Baulac \& Samson, 2011), por lo cual, el recuerdo de incidentes autobiográficos estaría afectado sin importar la lateralización de la ZII, puesto que ambos hipocampos podrían tener un funcionamiento deficiente en cada paciente (Gregory et al., 2015).

Se exploró la correlación entre la edad de inicio de la epilepsia y las posibles alteraciones en la MA. En memoria semántica personal, no se encontró ningún tipo de correlación. Tampoco se observó un mayor déficit en el recuerdo de incidentes autobiográficos en los pacientes con edad de inicio más temprana. Resultados similares ya han sido reportados. Viskontas, McAndrews y Moscovitch (2000), no encontraron correlación entre la edad de inicio de las crisis y el desempeño en incidentes autobiográficos, no hubo diferencia entre los pacientes que comenzaron las crisis antes de los 5 años o después de los 18 . De igual forma, no se encontró correlación entre la edad de inicio de las crisis y el recuerdo de incidentes autobiográficos en la investigación de Voltzenlogel, Després y Vignal (2006).

Hallazgos contrarios, han sido de igual forma informados. Lah, Lee, Garyson \& Miller (2006), quienes encontraron mayores alteraciones en el recuerdo de incidentes autobiográficos cuando la enfermedad inició antes de los 14 años. Para otros autores, la edad de inicio de las crisis no sería posible correlacionarla con los déficits cognitivos encontrados en los pacientes con ELT, ya que se ha afirma, que la $\mathrm{EH}$ se presenta varios años antes del inicio de las crisis, por lo que 
Julián Carvajal-Castrillón, Daniel Camilo Aguirre-Acevedo, David Andrés Montoya Arenas.

sería difícil especificar en qué edad se presentó la lesión cerebral que es causante de las crisis (Aikia, Salmenpera, Partanen, Kalviainen, 2001; Helmstaedter \& Elger, 2009; Stefan \& Pauli, 2002).

\section{Conclusiones}

Se encontró correlación entre el tiempo de evolución de la enfermedad y el bajo desempeño en memoria semántica personal. Este resultado sugiere que a mayor tiempo de evolución de la enfermedad, peor es el rendimiento de los pacientes con ELT por EH en la memoria personal semántica para el periodo de la infancia. Respecto al tratamiento, los pacientes con ELT por $\mathrm{EH}$ intervenidos con politerapia, presentan un desempeño significativamente inferior en la memoria semántica personal de la época de la escuela (6-11 años). Así mismo, se identificó una correlación entre el número de medicamentos y la MA. Los pacientes que empleaban más medicamentos, tuvieron mayores dificultades en memoria semántica personal para los periodos de la escuela (6-11 años), su boda y la vida reciente; así mismo, presentaron mayor afectación en el recuerdo de incidentes autobiográficos relacionados con la vida reciente (últimos 5 años).

Acerca de la edad de inicio de las crisis y el desempeño cognitivo, no se encontró correlación con la memoria personal semántica y recuerdo de incidentes autobiográficos. Finalmente, teniendo en cuenta la lateralización de la zona lesional, no se encontraron diferencias en el desempeño de los pacientes con ELT izquierdo en comparación ELT derecho, para ninguna de las dos dimensiones de la MA.

\section{Recomendaciones}

Los presentes resultados deben analizarse cuidadosamente, ya que se realizaron comparaciones múltiples, y la probabilidad de encontrar un resultado espúreo se incrementa por el número de contrastes estadísticos. Además, es importante mencionar que para futuros estudios que pretendan un mayor control de la tasa de error tipo I, el valor p que debe asumirse es $0.05 / \mathrm{k}$ donde $\mathrm{k}$ es el número de pruebas estadísticas realizadas.

\section{Referencias}

Aikia, M., Salmenpera, T., Partanen, K. \& Kalviainen, R. (2001). Verbal memory in newly diagnosed patients and patients with chronic left temporal lobe epilepsy. Epilepsy Behav, 1, 20-7. doi: 10.1006/ ebeh.2000.0140

Antérion, C., Mazzola, L., \& Laurent, B. (2008). Autobiographic memory:

Phenomenological aspects, personal semantic knowledge, generic events and characters (one case of pure retrograde memory recovery). Clinical Neurophysiology, 38, 171-6. doi: 10.1016/ j.neucli.2008.02.006

Araújo, D., Santos, A.C., Velasco, T.R., Wichert-Ana, L., Terra-Bustamante, V.C., Alexandre, V.Jr, Carlotti C.G. Jr., Assirati, J.A. Jr., Machado, H.R., Walz, R., Leite, J.P. \& Sakamoto, A.C. (2006). Volumetric evidence of bilateral damage in unilateral mesial temporal lobe epilepsy. Epilepsia, 47, 1354-9. doi: 10.1111/ j.1528-1167.2006.00605.x

Beghi, E., Berg, A., Carpio, A., Forsgren, L., Hesdorffer, D.C., Hauser, W.A ... Tomson, T. (2005). Epileptic seizures and epilepsy: definitions proposed by the International League Against Epilepsy (ILAE) and the International Bureau for Epilepsy (IBE). Epilepsia, 46, 470-2. doi: 10.1111/ j.0013-9580.2005.66104.x

Butler, C., Kapur, N., Zeman, A., Weller, R. \& Connelly, A. (2012). Epilepsy-related longterm amnesia: Anatomical perspectives. Neuropsychologia, 50, 2973-80. doi: 10.1016/j.neuropsychologia.2012.07.027

Carrillo-Mora, P. (2010). Sistemas de memoria: reseña histórica, clasificación y conceptos actuales. Segunda parte: Sistemas de memoria a largo plazo: Memoria episódica, 
sistemas de memoria no declarativa y memoria de trabajo. Salud mental, 33, 197-205.

Carvajal-Castrillón, J. \& Montoya, D.A. (2016). Memoria autobiográfica en epilepsia del lóbulo temporal por esclerosis hipocampal. Acta Neurol Colomb, 32, 100-7

Carvajal-Castrillón, J., Andrade, R., AguirreAcevedo, D.C. \& Montoya, D.A. (2016). Memoria autobiográfica en pacientes con epilepsia del lóbulo temporal. Revista CES psicología, 8, 200-12

Cendes, F. (2004). Febrile seizures and mesial temporal sclerosis. Curr Opin Neurol, 17, 161-4.

Cormack, F., Cross, J.H., Isaacs E., Harkness, W., Wright, I., Vargha-Khadem, F. \& Baldeweg, T. (2007). The Development of intellectual abilities in pediatric temporal lobe epilepsy. Epilepsia, 48, 201-4. doi: 10.1111/j.1528-1167.2006.00904.x

Feria-Romero, I.A., Alonso-Vanegas, M.A., Rocha-Arrieta, L., Villeda-Hernández, J., Escalante-Santiago, D., LorigadosPedré, L., Morales-Chacón, L., GrijalvaOtero, I. \& Orozco-Suárez, S. (2013). Mecanismos de neurodegeneración en la epilepsia del lóbulo temporal. Rev Chil Neuro-Psiquiat, 51, 137-48. doi: S0717-92272013000200007

Fritz, C. O., Morris, P. E., \& Richler, J. J. (2012). Effect size estimates: current use, calculations, and interpretation. Journal of Experimental Psychology: General, 141(1), 2. doi: $10.1037 / \mathrm{a} 0024338$

García, G., Contador, I., Fernández-Calvo, B. \& Ramos, F. (1989). Entrevista de memoria autobiográfica. Londres: Pearson.

Gregory, A.M., Nenert, R., Allendorfer, J.B., Martin, R., Kana, R.K. \& Szaflarski, J.P. (2015). The effect of medial temporal lobe epilepsy on visual memory encoding. Epilepsy Behav, 46, 173-84. doi: 10.1016/ j.yebeh.2015.03.006.

Helmstaedter, C. \& Elger, C.E. (2009). Chronic temporal lobe epilepsy: a neurodevelopmental or progressively dementing disease? Brain, 132, 2822-30. doi: 10.1093/brain/awp182

Helmstaedter, C., Elger, C.E. \& Witt, J.A. (2016). The effect of quantitative and qualitative antiepileptic drug changes on cognitive recovery after epilepsysurgery. Seizure, 36, 63-9. doi: 10.1016/j.seizure.2016.02.001.

Herfur, K., Kasper, B., Schwarz, M., Stefan, H. \& Pauli, E. (2010). Autobiographical memory in temporal lobe epilepsy: role of hipocampal and temporal lateral structures. Epilepsy Eु Behavior, 19, 365-71. doi: 10.1016/j.yebeh.2010.07.012

Kambaja, B. \& Dupont, S. (2016). Amnesia, memory disturbances and epilepsy. Geriatr Psychol Neuropsychiatr Vieil., 1, 415-419. doi: 10.1684/pnv.2016.0633

Keary, T.A, Frazier, T.W., Busch, R.M., Kubu, C.S. \& Iampietro, M. (2007). Multivariate neuropsychological prediction of seizure lateralization in temporal epilepsy surgical cases. Epilepsia, 48, 1438-46. doi: 10.1111/ j.1528-1167.2007.01098.x

Lah, S., Lee, T., Grayson, S. \& Miller, L. (2006). Effects of temporal lobe epilepsy on retrograde memory. Epilepsia, 47, 615-25. doi: $10.1111 / j .1528-1167.2006 .00476 . x$

Larner, A.J. (2014). Effect Size (Cohen's d) of Cognitive Screening Instruments Examined in Pragmatic Diagnostic Accuracy Studies. Dement Geriatr Cogn Disord Extra, 4, 236-41.

Lecrubier, Y. (1998). Mini Entrevista Neuropsiquiátrica Internacional (MINI). Versión de la CIE-10.

Marín, E., \& Ruiz, J. (2011). Memoria y metamemoria durante la amnesia global transitoria: estudio comparativo sobre su evolución a largo plazo. Rev Neurol, 53, $15-21$

Mohanraj, R. \& Brodie, M.J. (2006). Diagnosing refractory epilepsy: response to sequential treatment schedules. Eur J Neurol, 13, 277-82. doi: 10.1111/ j.1468-1331.2006.01215.x

Mula, M. \& Trimble, R. (2006). Neuropharmacological aspects of cognitive 
Julián Carvajal-Castrillón, Daniel Camilo Aguirre-Acevedo, David Andrés Montoya Arenas.

neurorehabilitation in epilepsy. Behavioral Neurology, 17, 69-75

Mula, M. \& Trimble, R. (2009). Antiepileptic drug-induced cognitive adverse effects: potential mechanisms and contributing factors. CNS, 23, 121-37. doi: 10.2165/00023210-200923020-00003.

Múnera, C.P., Lomlomdijan, C., Gori, B., Terpiluk, V., Medel, N., Solis, P. \& Kochen, S. (2014). Episodic and semantic autobiographical memory in temporal lobe epilepsy. Epilepsy Research and Treatment, 1-9. doi: $10.1155 / 2014 / 157452$

Nadel, L., Samsonovich, A., Ryan, L. \& Moscovitch, M. (2000). Multiple trace theory of human memory: computational, neuroimaging, and neuropsychological results. Hippocampus, 10, 352-68. doi: 10.1002/1098-1063(2000) 10:4<352::AID$\mathrm{HIPO}>3.0 . \mathrm{CO} ; 2-\mathrm{D}$

Narayanan, J., Duncan, R., Greene, J., Leach, J.P., Razvi, S., McLean, J. \& Evans, J. (2012). Accelerated long-term forgetting in temporal lobe epilepsy: Verbal, nonverbal and autobiographical memory. Epilepsy E) Behavior, 25, 622-30. doi: 10.1016/ j.yebeh.2012.06.038

Nunes, J.C., Zakon, D.B., Claudino, L.S., Guarnieri, R., Bastos, A., Queiroz, L.P., Walz, R. \& Lin, K. (2011). Hippocampal sclerosis and ipsilateral headache among mesial temporal lobe epilepsy patients. Seizure, 20, 480-4. doi: 10.1016/j.seizure.2011.02.014

Pfeuty, M., Thivard, L., Dupont, S., Adam, C., Baulac, M. \& Samson, S. (2011). Postoperative recovery of hippocampal contralateral diffusivity in medial temporal lobe epilepsy correlates with memory functions. J Neurol Neurosurg Psychiatry, 82, 340-3. doi: 10.1136/jnnp.2008.158428

Sheth, R.D. (2002). Epilepsy surgery. Presurgical evaluation. Neurol Clin, 20, 1195-215.

Stefan, H. \& Pauli, E. (2002). Progressive cognitive decline in epilepsy: an indication of ongoing plasticity. Prog Brain Res, 135, 409-17. doi: 10.1016/ S0079-6123(02)35038-6
St-Lauren, M., Moscovich, M., Levine, B. \& McAndrews, M.P. (2009). Determinants of autobiographical memory in patients with unilateral temporal lobe epilepsy or excisions. Neuropsychologia, 47, 2211-21. doi: 10.1016/j.neuropsychologia.2009.01.032

Uscátegui, A.M. \& Izquierdo, A. (2003). Esclerosis mesial temporal. Acta Neurol Colomb, 19, 203-8.

Viskontas, I.V., McAndrews, M.P. \& Moscovitch, M. (2000). Remote episodic memory deficits in patients with unilateral temporal lobe epilepsy and excisions. The Journal of Neuroscience, 20, 5853-7.

Voltzenlogel, V., Després, O., Vignal, J.P., Steinhoff, B., Kehrli, P. \& Manning, L. (2006). Remote Memory in Temporal Lobe Epilepsy. Epilepsia, 47, 1329-36. doi: 10.1111/j.1528-1167.2006.00555.x

Voltzenlogel, V., Vignal, J.P., Hirsch, E. \& Manning, L. (2014). The influence of seizure frequency on anterograde and remote memory in mesial temporal lobe epilepsy. Seizure, 23, 792-8. doi: 10.1016/ j.seizure.2014.06.013.

Wechsler, D. (1997). Wechsler Adult Intelligence Scale III. San Antonio: Psychological Corporation.

Wiebe, S. (2000). Epidemiology of temporal lobe epilepsy. Can J Neurol Sci, 27, S6-10.

\section{Notas}

* Artículo de investigación. 